\title{
Color Image Enhancement Algorithms based on the DCT Domain
}

\author{
Junjun Xia, Karen Panetta, IEEE Fellow \\ Dept. of Electrical and Computer Engineering \\ Tufts University \\ Medford, MA \\ Junjun.Xia@tufts.edu, Karen@ece.tufts.edu
}

\author{
Sos Agaian, Senior Member \\ Dept. of Electrical and Computer Engineering \\ University of Texas at San Antonio \\ San Antonio, TX \\ Sos.Agaian@usta.edu
}

\begin{abstract}
-this paper presents a novel modified multi scale contrast enhancement (MMCE) technique for color image enhancement based on manipulating the DCT coefficients. Modified multi contrast enhancement (MCE) is an improved version of multi contrast enhancement by redefining the frequency spectral bands and introducing more band enhancement techniques to achieve a better performance. This paper also uses an image contrast measure SDME to choose the optimal parameters and to demonstrate the effectiveness of the methods. Computer simulations and analysis are shown that the presented method outperforms the commonly used methods such as Retinex and the original MCE for most images.
\end{abstract}

Keywords- alpha rooting; logarithmic enhancement; multiscale contrast enhancement; color image enhancement ;

\section{INTRODUCTION}

Image enhancement is used to visually improve the quality of processed images, by typically focusing on expanding the dynamic range and improving the image contrast. Several enhancement algorithms have been proposed both in spatial domain and transform domain. Spatial domain enhancement algorithms are directly applied on the pixel value. Most of these methods are based on histogram modification. The transform domain methods first transform the gray level image into the frequency domain by using the Cosine, Fourier, or Wavelet transforms and then modify the transform domain coefficients. The advantages of transform domain techniques are that they do not affect the ability to compress the original image, require less memory space and a lower computational cost as compared to spatial domain techniques [1]. Tang and Peli [2-3] proposes a contrast definition of DCT coefficients which is the ratio of high frequency content and low frequency content in the bands of $8 * 8$ DCT matrix and enhanced the contrast by multiplying a user controlled constant factor. Peli's method in [4] proposes a modified constant measure which changes the constant factor to a function and defines different function for the high frequency and low frequency coefficient. Lee [5] combines the basic concept of retinex which is used to modify the DC coefficients and along with using multiscale contrast enhancement. This results in simultaneously compressing the image's dynamic range and enhancing the image's contrast. However, the transform domain methods often introduce block artifacts and superfluous edges near block boundaries. There are other limitations such as they cannot simultaneously enhance all portions of the image very well and selection of the optimal parameters still posses a critical issue for automating the process. [6]

Usually in color image enhancement, the color images are transformed into another color space where chromatic components are more uncorrelated from the achromatic component, such as $\mathrm{HSV}, \mathrm{YCbCr}$ and then apply the gray image enhancement algorithm to the luminance component. In [7], a modified PCA color space which replaces the mean operation with the median operation was proposed. It can introduce fewer artifacts since the median operation truncates the floating point values resulting in efficiently protecting the edge information. There are also some color image enhancement algorithms proposed which work directly on the RGB color space, such as retinex techniques [8] and 3D histogram equalization. [9]

This paper proposes an improved version of the multiscale contrast enhancement algorithm by redefining the frequency spectral bands and including with more enhancement techniques for the bands. This paper will also demonstrate a quantitative enhancement measurement based upon contrast entropy to choose the optimal algorithm parameters and determine the effectiveness of the method. The paper is organized as follows: Section II provides the relative background knowledge of the presented algorithm. Section III presents the new image enhancement algorithm based on modifying the original Multiscale contrast enhancement algorithm. Section IV is computational simulation results of the presented algorithm and a comprehensive comparison is made to demonstrate the effective of the proposed algorithm. Section $\mathrm{V}$ provides a discussion of color image enhancement and some concluding comments are made.

\section{BACKGROUND}

In this section, we will cover the background topics necessary to understand the proposed methods. First we give a brief introduction of alpha rooting and logarithmic enhancement techniques, which will be used in the presented 
algorithms. We then present an image measure SDME [10] which aids in the optimal parameter selection in the algorithm.

\section{A. Alpha-rooting[1]}

Alpha rooting can be described through the following block diagram:

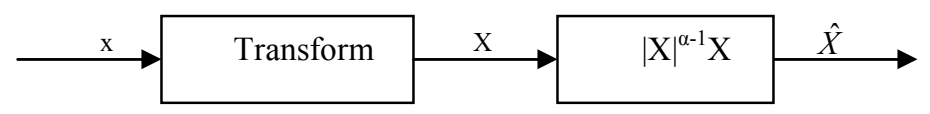

Fig. 1: diagram of $\alpha$-rooting

The block diagram shows the magnitude of each transformed coefficient is raised to power $\alpha$, where $0<\alpha<1$ and the sign or phase of the coefficient is unchanged $\hat{X}\left(\mathrm{n}_{1}, \mathrm{n} 2\right)$ can be written as

$$
\hat{X}\left(n_{1}, n_{2}\right)=\left|X\left(n_{1}, n_{2}\right)\right|^{\alpha-1} X\left(n_{1}, n_{2}\right)
$$

When $\alpha$ is equal to zero, we get the sign of the coefficients. With $0<\alpha<1$, the amplitude of the large transform coefficients is reduced more relative to the small transform coefficients. Since high frequencies are often associated with small transform coefficients, they contain all the pertinent edge and detailed information for the enhancement algorithm.

\section{B. Logarithmic enhancement [13]}

Logarithmic enhancement was introduced by Agaian in [13]. This method reduced the transform coefficient magnitudes while leaving the phase unchanged, using a logarithmic rule. Logarithmic enhancement uses two parameters, namely, $\beta$ and $\lambda$. It works according to the following transfer function.

$$
O(p, s)=\log ^{\beta}\left(|X(p, s)|^{\lambda}+1\right)
$$

Both alpha rooting and logarithmic enhancement can be used in combination with many different orthogonal transforms such as the Fourier, Hartley and cosine transforms. The resulting output shows an emphasis on the high frequency content of the image without changing the phase of the transform coefficients, resulting in an overall contrast enhancement.

\section{SDME [10]}

Since image enhancement is intended to improve the contrast, the enhancement measure is usually based on contrast measure. To quantitatively assess the enhancement performance of the presented algorithm, we use an enhancement measure using an image contrast measure based on the concept of the second derivative [10].It is called the second-derivative-like measure of enhancement (SDME) which is defined by,

$$
S D M E=-\frac{1}{k_{1} k_{2}} \sum_{l=1}^{k_{1}} \sum_{k=1}^{k_{2}} 20 \ln \left|\frac{I_{\max ; k, l}-2 I_{\text {center } ;, l}+I_{\min ; k, l}}{I_{\max ; k, l}+2 I_{\text {center } ; k, l}+I_{\min ; k, l}}\right|
$$

where an image is divided into $k_{1} \times k_{2}$ blocks. $I_{\text {max; } ;, l}, I_{\text {min }, k, l}$ are the maximum and minimum values of the pixels in each block separately, and $I_{\text {center } ; k, l}$ is the center pixel value in each block. Thus, the size of blocks should be odd number such as $3 \times 3$. Each block contains an odd number of pixels.

\section{METHODOLOGY}

The display of a color image depends upon three fundamental factors, brightness, contrast and color. The luminance component contains the largest values of the components in PCA. The other two components of PCA are chrominance information. Luminance and chrominance are processed in parallel and the luminance is first treated by Gamma correction to adjust the background brightness and then the proposed algorithm which is used to improve image contrast. The final step is color preservation. The chrominance components are weighted by a constant factor in order to compensate for the loss of saturation induced by the increase in luminance. The diagram is shown on Fig2.

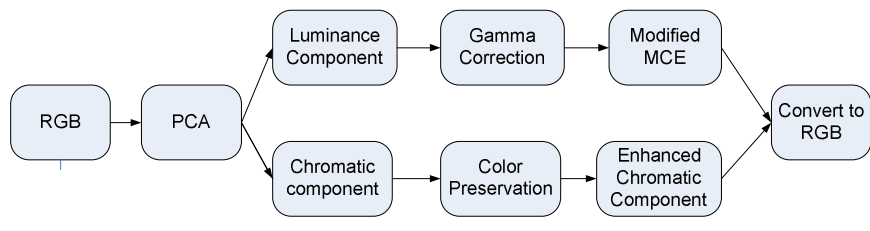

Fig. 2: Diagram of the color enhancement histogram shifting algorithm

\section{A. Dynamic range adjustment}

The purpose of dynamic range compression is to map the natural dynamic range of a signal to a smaller range. In the presented algorithm, it is achieved by Gamma Correction. We refine the illumination image by Gamma correction operation with a free parameter $\gamma$.The Gamma correction is performed by following formula:

$$
Y^{\prime}=W\left(\frac{Y}{W}\right)^{1 / \gamma}
$$

Where $\mathrm{W}$ is the white value (equal to 255 in 8 bit images).

According to the characteristics of the mapping function, the range of the suitable enhancement parameter should be centered around two. The value should be further adjusted according to the contents and brightness of the input image.

\section{B. Modified multi contrast enhancement (MMCE)}

Multi contrast enhancement is a direct contrast enhancement algorithm based on a definition of image contrast in the DCT domain. The novelties of this method lie in spectral band division and a definition of contrast based on transform coefficient. First, the $8 * 8$ array of DCT coefficients are classified into 15 different frequency bands. The nth band 
is composed of the coefficients with $n=k+1$. A band defined by $\mathrm{n}=\mathrm{k}+\mathrm{l}$ gives a diamond-shaped approximation to a circle and thus, selects approximately equal radial frequencies. As the band number increases, the frequency content of the bandpass image block corresponds with higher frequencies and creates a primitive multiscale structure. [2-3]

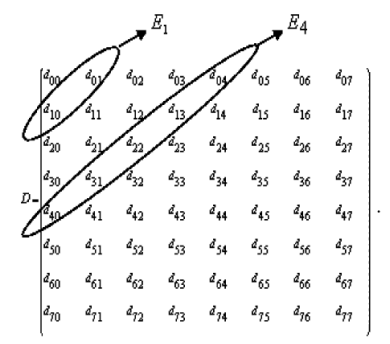

Fig. 3: Bands in Cosine Transform Matrix

The proposed algorithm improves multi contrast enhancement in two respects: 1 Redefine the frequency spectral band. 2 Enhance each band coefficients by including more enhancement techniques such as alpha rooting, logarithmic enhancement in addition to the traditional scaling. In [2-3], a band of DCT coefficients is defined by $\mathrm{n}=\mathrm{k}+\mathrm{l}$ which shows a diamond-shaped approximation to a circle and has approximately equal radial frequencies, shown on Fig (3). In the paper, we propose three new different band division strategies.

1. Each element is a band, which means we do not classify any elements into a band and it becomes a global enhancement techniques for each block.

2. The second band definition is shown on Fig4 (a). It classifies the entire frequency domain into 5 spectral bands.

$E_{0}$ is the low frequency band and $E_{1}, \ldots . E_{5}$ are the high frequency bands, which is shown Fig 4 . The benefits of this band division are that the low frequency band $E_{0}$ contain 9 low frequency coefficients and we can treat this band with a smaller factor to aid in reducing the blocking artifacts.

3. In our algorithm, we use this band division definition, which is shown on Fig4 (b). From Fig4 (b), we can see that we define each column and each row as a separate band. The column bands are $E_{c 0}, E_{c 1}, \ldots . E_{c 7}$.The row bands are $E_{r 0}, E_{r 1}, \ldots . E_{r 7}$. Based on the characteristic of DCT coefficients, this band definition is especially useful for edge enhancement. For example, to enhance the horizontal edge, the column bands should be treated, since the horizontal edge information lies in the vertical DCT coefficients, while for vertical edges, the row bands should be enhanced.

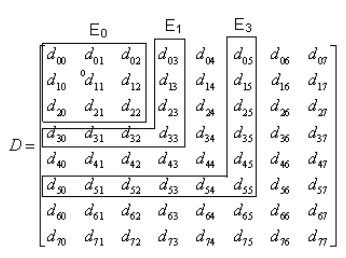

(a)

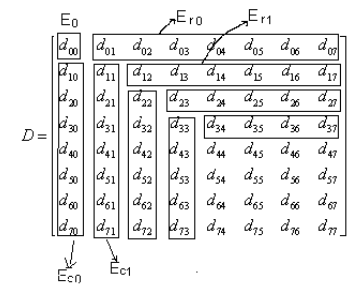

(b)
Fig. 4: Proposed Bands divisions of DCT coefficients

The MCE method only modifies the DCT coefficients by scaling. Another improvement of the presented MMCE algorithm is introducing some other enhancement techniques into the process that offer more possibilities to modify the coefficients instead of only scaling., These methods include alpha rooting and logarithmic enhancement. Furthermore, for different bands we can use different parameter values.

The energy of each band is defined based on the enhanced $X^{\prime}$

$$
E_{t}=\frac{\sum_{(i, j) \in \text { band }}|f(d(i, j))|}{N}
$$

where $\mathrm{N}$ is the total number of coefficients in the band, we have several possibilities for function $f\left(d_{i, j}\right)$ which offers greater flexibility. The function can be of the forms:

a) $f(d(i, j))=d(i, j)^{\alpha_{t}}$, which is alpha rooting

b) $f(d(i, j))=\log ^{\beta_{n}}(d(i, j))$, which is the logarithmic enhancement technique

Where $d(i, j)$ belong to the same frequency band

For column bands, $E_{c t}, j=t, i=t . .7$

And for row bands, $E_{r t}, i=t, j=t \ldots 7$

The enhancement parameters $\alpha$ and $\beta$ can be adaptive for different bands. In our implementation, we take them as constants for all bands.

The following scaling process is the same as the MCE method, the enhanced contrast is defined as

$$
C_{n}^{\prime}=\lambda C_{n}
$$

Where

$$
C_{n}=\frac{E_{n}}{\sum_{t=0}^{n-1} E_{t}}
$$

Equation (6) can be stated as

$$
E_{n}^{\prime}=\lambda H_{n} E_{n}
$$


where $H_{n}=\frac{\sum_{t=0}^{n-1} E_{t}^{\prime}}{\sum_{t=0}^{n-1} E_{t}}$

The pseudo code for the proposed algorithm is as follows: Step0: Divide the image into $8 * 8$ blocks and apply the DCT to each block. For each block do steps 1 through 3 .

Step1: Treat the low frequencies by a smaller weighting factor $\lambda$, usually set to 1.2 .

Step2: For the high frequency component enhancement, first choose the enhancement function and then, based on the measure select the optimal parameter. Finally, recursively compute $\mathrm{H}$ and enhance all the high frequency DCT coefficients.

Step3: When all bands have been enhanced, apply the inverse DCT.

Step4: Reassemble all the enhanced blocks together to obtain the final resulting enhanced image.

The SDME measure is not only a tool for objectively judging the enhancement performance, but also provides a mathematical tool for automatically choosing the optimal parameters. By plotting the SDME value of the enhanced image versus the parameters for a specific image, we can return a descriptive graph to help choose parameters, which is shown in Fig 6 We determine the optimal parameters by looking at the graph for the local extremum, and if none exists, we have to choose it by trial and test. As the parameter values get larger, so does the SDME. However, the image quality begins to decrease. To avoid the over enhancement, we need to decide suitable parameter values based on the SDME value and visual judgment together. Table 1 and Table 2 show the optimal parameter values based on the SDME value.

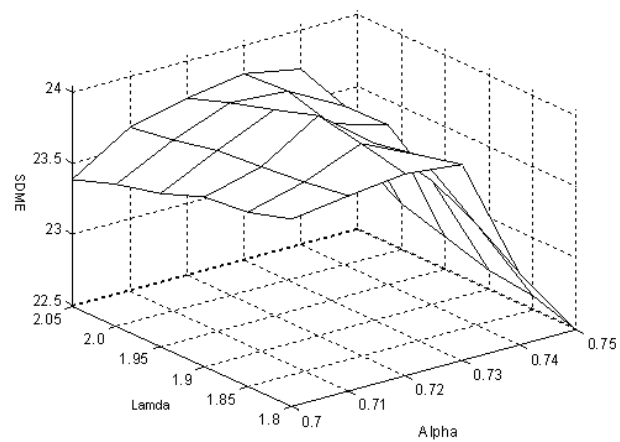

Fig.5: SDME VS parameter $\lambda$ and $\alpha$ for MMCE based on aplpha rooting

Table 1 The optimal parameters for MMCE based on alpha rooting

\begin{tabular}{|c|c|c|}
\hline SDME & $\lambda$ & $\alpha$ \\
\hline 23.76 & 1.92 & 0.72 \\
\hline 23.5 & 2.1 & 0.701 \\
\hline
\end{tabular}

Table 2 The optimal parameters for MMCE based on logarithmic enhancment technique

\begin{tabular}{|c|c|c|}
\hline SDME & $\lambda$ & $\beta$ \\
\hline 23.01 & 1.89 & -0.31 \\
\hline 21.05 & 1.7 & -0.22 \\
\hline
\end{tabular}

Fig.6 is an example which clearly shows the benefits of the presented algorithm. Fig7 (e) and (g) are the enhancement results by proposed method, which efficiently retains the details and improves the contrast and has a better visual quality than original image. Notice that it especially contains less block artifacts. Comparing with (c), which is the enhanced result by original MCE, they both have wider histograms thus expanding the dynamic range.

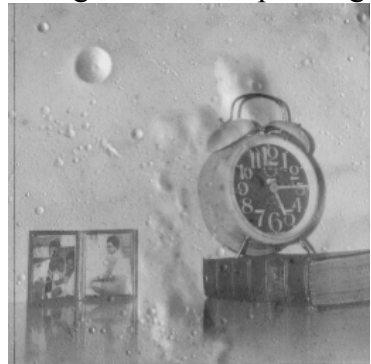

(a) $\mathrm{SDME}=19.38$

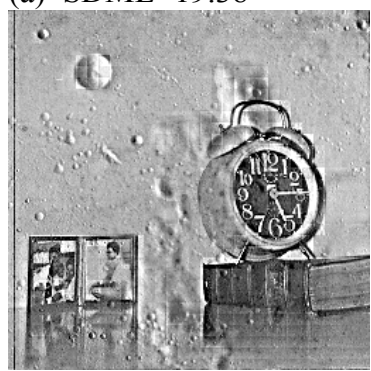

(c) $\mathrm{SDME}=22.08$

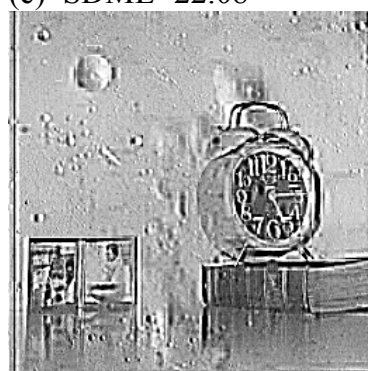

(e) $\mathrm{SDME}=23.76$

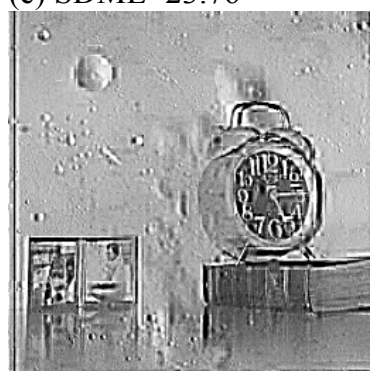

(g) $\mathrm{SDME}=23.01$

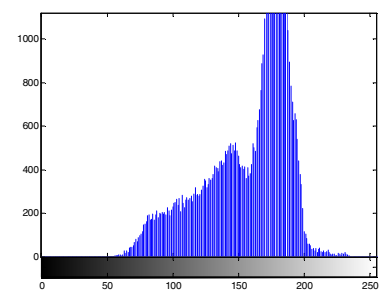

(b)

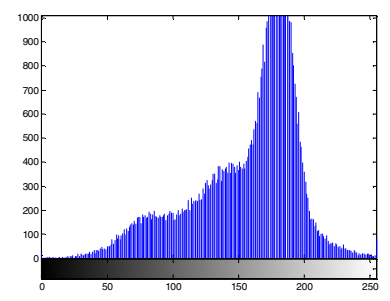

(d)

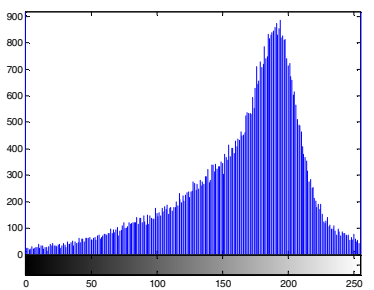

(f)

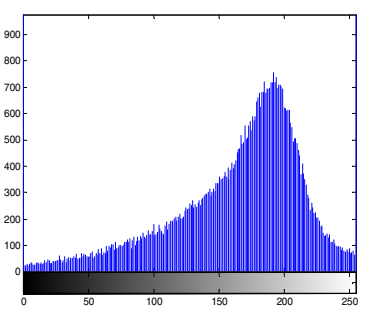

(h) 
Fig. 6: Enhancement results. (a) Original image and (b) is the histogram of (a). (c) is the enhanced results by multi scale enhancement and (d) is the histogram of (b). (e) is the enhanced result by proposed method based on alpha rooting and (f) is the histogram of (e). (g) is the enhanced result by proposed method based on logarithmic enhancement technique and $(\mathrm{h})$ is the histogram of $(\mathrm{g})$.

\section{Color preservation}

After the enhancement of the illumination component, the final step is to preserve the color. Although in the above color space the chrominance components are decorrelated much better than that in the RGB color space, only changing the luminance component and keeping the chrominance components unaltered cannot preserve all the color information. The increasing of illumination value would desaturate the color information. By preservation of colors, we multiply constant factors (color preservation factor) to compensate the desaturated effect on chromatic components.

$$
\begin{aligned}
& C_{1}^{\prime}=\alpha_{1} C_{1} \\
& C_{2}^{\prime}=\alpha_{2} C_{2}
\end{aligned}
$$

The constant factors are chose by trial and error. For different images, different color spaces, these values are different; usually the range of the value is between 1 and 4 . The improvement of the quality of the enhanced image is quite apparent from Fig. 7 .
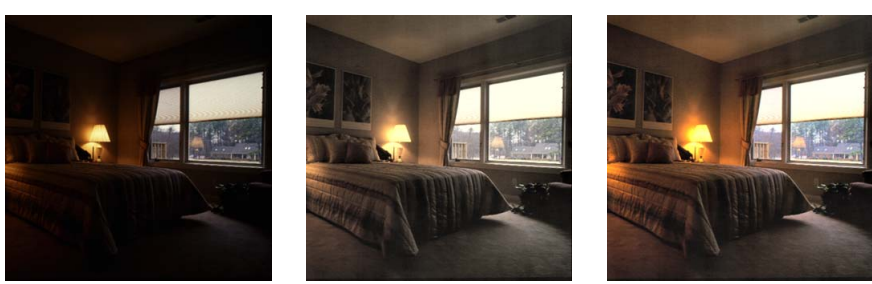

Fig.7. Enhanced image by processing only the illumination component and both the illumination and chrominance component on the image girl.

\section{COMPUTER SIMULATIONS}

This section performs a series of experiment results to demonstrate the performance of the proposed algorithms: modified Multiscale contrast enhancement (MMCE) based on alpha rooting and the modified Multiscale contrast enhancement based on logarithmic enhancement. We compare with three existing color enhancement algorithms, namely multi scale color image enhancement with color restoration (MSRCR),[10] Multiscale contrast enhancement (MCE) [3] , and alpha rooting. MCE and alpha rooting are proposed for gray image enhancement. For the color comparison, we extend it to color image enhancement based on the PCA color space. Luminance enhancement is performed by MCE and color preservation is achieved by our color preservation method. The MSRCR images were obtained with the free version of the software "Photo Flair" using the default setting [11], which put "demo" tags across the image. Figure 9 shows the comparison results. Table 2 shows the

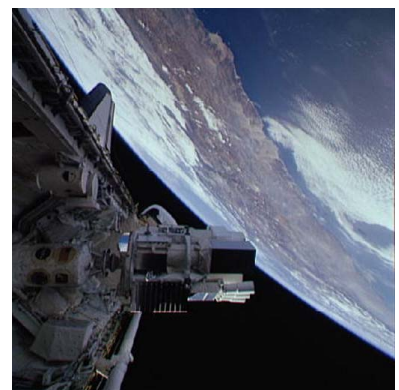

(a) original image $\mathrm{SDME}=35.02$

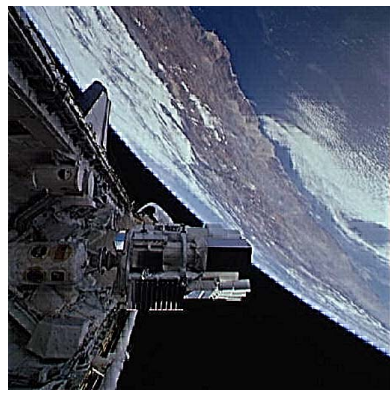

(c) enhanced by MCE $\mathrm{SDME}=36.15$

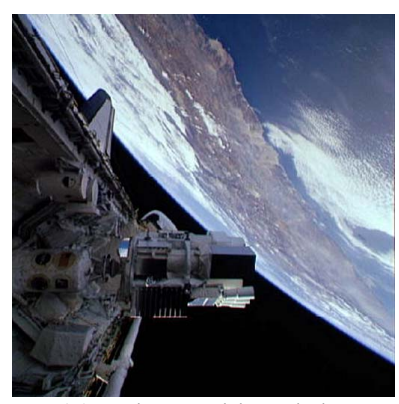

(e) enhanced by alpha rooting $\mathrm{SDME}=35.22$

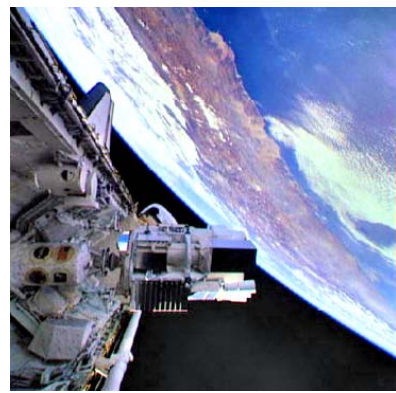

(b)enhanced by MMCE based on alpha rooting $\mathrm{SDME}=37.02$

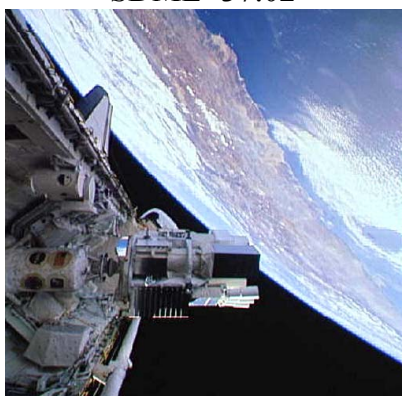

(d)enhanced by MMCE based on logarithmic enhancement $\mathrm{SDME}=39.22$

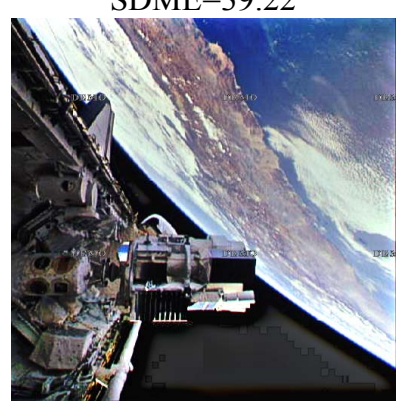

(f) enhanced by MSRCR $\mathrm{SDME}=22.03$
Fig.8. Examples of enhanced results of all the algorithms.

The benefits of the presented algorithms are clearly seen in all the images. The MMCE based on alpha rooting provides the good color rendition, brightness adjustment and contrast enhancement. With MCE, although the contrast has been improved very well, the enhanced image has serious block artifacts and some false edge information, also the brightness of image was not increased. Comparing with MCE, the MMCE algorithm contains two crucial steps: brightness adjustment which is achieved by Gamma correction and block artifacts suppression which obtained by redefining the frequency spectral band and differently treating high frequency coefficient and low frequency coefficients and keep low frequency coefficient relatively stable. Also by 
introducing other enhancement techniques such as alpha rooting and logarithmic enhancement, it can provide more balanced results than individual techniques. It outperforms the original MCE, which is reflected by better brightness and fewer artifacts. The enhanced images by alpha rooting only show a minimal improvement on the contrast and show no significant difference than the original images. All of the above algorithms are based the DCT domain except for MSRCR, which is a spatial domain enhancement algorithm. It has good performance with respect to color rendition and contrast enhancement; however, it cannot efficiently adjust the luminance range and has high computational complexities. The SDME values, which are the measure of luminance component, also show that the MMCE algorithms provide the better contrast enhancement than other algorithms.

\section{CONCLUSIONS}

This paper presents new techniques for color enhancement based on the manipulation of the DCT coefficients. The modified multiscale contrast enhancement (MMCE) treats low frequency and high frequency coefficients differently to suppress the block artifacts and protect large edge information in the low frequency components. By introducing alpha rooting and logarithmic enhancement function to the band enhancement, it provides high flexibility to modify the DCT coefficients. It provides more balanced results than applying the individual techniques alone. To adjust the brightness, Gamma correction is applied to some images. A compensation factor to the chrominance component is applied to preserve color information. A comparative study with different algorithms was conducted. The investigation includes mutiscale retinex with color restoration, multi contrast enhancement and alpha rooting. Computer simulations and analysis were provided to compare the enhancement performance. The experimental results and analysis show that the proposed algorithms are simple, fast and efficient color image enhancement algorithms which outperform other methods for most images. Also due to the simplicity, they show promise for to real time applications.

\section{ACKNOWLEDGMENT}

This work has been partially supported by NSF Grant HRD-0932339.

\section{REFERENCES}

[1] S. Agaian, B. Silver and K. Panetta, "Transform coefficient histogrambased image enhancement alogrithms usingcontrast entropy," in IEEE Transactions on Image Processing, vol.16,no.3,pp.741-758,2007

[2] J. Tang, E. Peli, and J. Kim, "Image enhancement in the JPEG domain for people with vision impairment, " IEEE Trans. Biomedical Engineering, vol. 51, pp. 2013-2023, November 2004.

[3] J. Tang, E. Peli, and S. Acton, "Image enhancement using a contrast measure in the compressed domain," IEEE Signal Processing Letters, vol. 10, pp. 289-292, October 2003.

[4] E.Vale and A.Alcaim "Image enhancement in the 2D DCT domain using a band adaptive contrast modification" IEEE ISCCP 1516-1519, Mar.2008

[5] S. Lee, "An efficient content-based image enhancement in the compressed domain using retinex theory," IEEE Trans. Circuits Syst. Video Technol., vol. 17, no. 2, pp. 199-213, Feb. 2007.

[6] S. Agaian, K Panetta, and A. Grigoryan, "A New Measure of Image Enhancement",IASTED International Conference on Signal Processing \& Communication, 19-22 Sept. 2000, Marbella, Spain.

[7] K.Panetta ,S. Qazi, S.Agian Techniques for Detection and Classification of Edges in Color Images Mobile Multimedia/Image Processing, Security, and Applications 2008Proc. SPIE, Vol. 6982, 69820W (2008)

[8] D. J. Jobson, Z. Rahman, and G. A. Woodell, "Properties and performance of a center/surround retinex," IEEE Transactions on Image Processing, vol.6, no. 3, pp. 451-462, Mar. 1997

[9] J. Han,S.Yang and B.Lee "a novel 3D color histogram equalization method with 1-D uniform gray scale histogram"," IEEE Transactions on Image Processing, vol.20, no. 20, pp. 506-512, Feb. 2011

[10] Y. Zhou, K. Panetta, and S. Agaian, "Mammogram Enhancement Using Alpha Weighted Quadratic Filter," in The $31^{\text {st }}$ Annual International Conference of the IEEE Engineering in Medicine and Biology Society, EMBS 2009, Minneapolis, Minnesota, 2009, pp. 3681-3684.

[11] http://trueview.com

[12] S. Agaian, K. Panetta, K. Grigoryan, Transform-based image enhancement algorithms with performance measure " IEEE Transactions on image processingVolume: 10, Issue: 32001 , Page(s): 367 - 382 